\title{
Corrigendum to "Low-Grade Endometrial Stromal Sarcoma with a Nodule-in-Nodule Appearance in Preoperative Magnetic Resonance Images"
}

\author{
Mitsuhiro Nakamura, ${ }^{1,2}$ Ryusuke Murakami $\mathbb{D}^{1,3}$ Kaoru Abiko, ${ }^{1,4}$ Taito Miyamoto $\mathbb{D},{ }^{1}$ \\ Yoshimi Kitawaki, ${ }^{1}$ Ken Yamaguchi, ${ }^{1}$ Akihito Horie, ${ }^{1}$ Junzo Hamanishi, ${ }^{1}$ Eiji Kondoh $\mathbb{D},{ }^{1}$ \\ Tsukasa Baba, ${ }^{1,5}$ Aki Kido, ${ }^{6}$ Sachiko Minamiguchi, ${ }^{7}$ Noriomi Matsumura $\mathbb{D}^{1,8}$ \\ and Masaki Mandai ${ }^{1}$ \\ ${ }^{1}$ Department of Gynecology and Obstetrics, Kyoto University Graduate School of Medicine, Kyoto, Japan \\ ${ }^{2}$ Department of Obstetrics and Gynecology, Japanese Red Cross Society Wakayama Medical Center, Wakayama, Japan \\ ${ }^{3}$ Department of Gynecology, Shiga General Hospital, Shiga, Japan \\ ${ }^{4}$ Department of Obstetrics and Gynecology, National Hospital Organization Kyoto Medical Center, Kyoto, Japan \\ ${ }^{5}$ Department of Obstetrics and Gynecology, Iwate Medical University Faculty of Medicine, Iwate, Japan \\ ${ }^{6}$ Department of Diagnostic Imaging and Nuclear Medicine, Kyoto University Graduate school of Medicine, Kyoto, Japan \\ ${ }^{7}$ Department of Diagnostic Pathology, Kyoto University Graduate School of Medicine, Kyoto, Japan \\ ${ }^{8}$ Department of Obstetrics and Gynecology, Kindai University Hospital, Osaka, Japan \\ Correspondence should be addressed to Ryusuke Murakami; ryusukem@kuhp.kyoto-u.ac.jp
}

Received 31 January 2021; Accepted 31 January 2021; Published 12 February 2021

Copyright (c) 2021 Mitsuhiro Nakamura et al. This is an open access article distributed under the Creative Commons Attribution License, which permits unrestricted use, distribution, and reproduction in any medium, provided the original work is properly cited.

In the article titled "Low-Grade Endometrial Stromal Sarcoma with a Nodule-in-Nodule Appearance in Preoperative Magnetic Resonance Images" [1], there was a spelling error in author Eiji Kondoh's name in the author list, where Eiji Kodoh should have read Eiji Kondoh. This is corrected as shown above.

\section{References}

[1] M. Nakamura, R. Murakami, K. Abiko et al., "Low-Grade Endometrial Stromal Sarcoma with a Nodule-in-Nodule Appearance in Preoperative Magnetic Resonance Images," Case Reports in Obstetrics and Gynecology, vol. 2020, Article ID 8973262, 7 pages, 2020. 